\title{
Inclusion of medication-related fall risk in fall risk assessment tool in geriatric care units
}

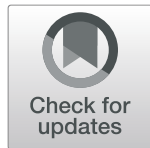

Jana Michalcova ${ }^{1,2^{*}}$ (D), Karel Vasut ${ }^{1}$, Marja Airaksinen ${ }^{2}$ and Katarina Bielakova ${ }^{3}$

\begin{abstract}
Background: Falls are common undesirable events for older adults in institutions. Even though the patient's fall risk may be scored on admission, the medication-induced fall risk may be ignored. This study developed a preliminary categorization of fall-risk-increasing drugs (FRIDs) to be added as a risk factor to the existing fall risk assessment tool routinely used in geriatric care units.

Methods: Medication use data of older adults who had experienced at least one fall during a hospital ward or a nursing home stay within a 2-year study period were retrospectively collected from patient records. Medicines used were classified into three risk categories (high, moderate and none) according to the fall risk information in statutory summaries of product characteristics (SmPCs). The fall risk categorization incorporated the relative frequency of such adverse drug effects (ADEs) in SmPCs that were known to be connected to fall risk (sedation, orthostatic hypotension, syncope, dizziness, drowsiness, changes in blood pressure or impaired balance). Also, distribution of fall risk scores assessed on admission without considering medications was counted.

Results: The fall-experienced patients ( $n=188,128$ from the hospital and 60 from nursing home records) used altogether 1748 medicaments, including 216 different active substances. Of the active substances, 102 (47\%) were categorized as high risk (category A) for increasing fall risk. Fall-experienced patients $(n=188)$ received a mean of 3.8 category A medicines $(n=710), 53 \%(n=375)$ of which affected the nervous and $40 \%(n=281)$ the cardiovascular system. Without considering medication-related fall risk, $53 \%(n=100)$ of the patients were scored having a high fall risk (3 or 4 risk scores).

Conclusion: It was possible to develop a preliminary categorization of FRIDs basing on their adverse drug effect profile in SmPCs and frequency of use in older patients who had experienced at least one documented fall in a geriatric care unit. Even though more than half of the fall-experienced study participants had high fall risk scores on admission, their fall risk might have been underestimated as use of high fall risk medicines was common, even concomitant use. Further studies are needed to develop the FRID categorization and assess its impact on fall risk.
\end{abstract}

Keywords: Older adults, Fall risk, Medication, Hospital, Nursing home, Preventive risk management

\footnotetext{
* Correspondence: jana.michalcova@icloud.com

${ }^{1}$ Faculty of Pharmacy, Department of Applied Pharmacy, Masaryk University,

Palackeho 1946/1, 61242 Brno, Czech Republic

${ }^{2}$ Faculty of Pharmacy, Division of Pharmacology and Pharmacotherapy,

Clinical Pharmacy Group, University of Helsinki, Viikinkaari 5E, 00014 Helsinki, Finland

Full list of author information is available at the end of the article
}

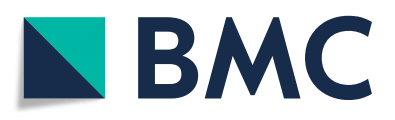

(- The Author(s). 2020 Open Access This article is licensed under a Creative Commons Attribution 4.0 International License, which permits use, sharing, adaptation, distribution and reproduction in any medium or format, as long as you give appropriate credit to the original author(s) and the source, provide a link to the Creative Commons licence, and indicate if changes were made. The images or other third party material in this article are included in the article's Creative Commons licence, unless indicated otherwise in a credit line to the material. If material is not included in the article's Creative Commons licence and your intended use is not permitted by statutory regulation or exceeds the permitted use, you will need to obtain permission directly from the copyright holder. To view a copy of this licence, visit http://creativecommons.org/licenses/by/4.0/. The Creative Commons Public Domain Dedication waiver (http://creativecommons.org/publicdomain/zero/1.0/) applies to the data made available in this article, unless otherwise stated in a credit line to the data. 


\section{Background}

Falls constitute a leading preventable cause of geriatric injuries and hospitalization, prolonged recovery times and deaths $[1,2]$. More than a third of older adults fall each year, meaning worldwide a high number of patients [3]. Fall-related injuries can be serious, occuring approximately in $10 \%$ of falls, and consequencing an urgent hospitalization [3, 4]. Furthermore, falls decrease self-sufficiency and quality of life among older adults, because falls can result in fear of falling, loss of confidence, mobility and ability to live independently [5].

Fall risk is a multifactorial problem associated with numerous intrinsic and extrinsic factors [6, 7]. Intrinsic factors are related to ageing, including age-related changes to the pharmacokinetic and pharmacodynamic effects of pharmacotherapies. Age-related pharmacokinetic changes are represented by drug absorption, distribution, metabolism and elimination route. The drug absorption is usually decreased due to changes in the gastrointestinal tract, e.g., reduced gastric secretion, loss of mucosal intestinal surface and decreased blood flow in splanchnic area. The drug distribution is affected by many factors, e.g., increase in fat compartment, decrease in total body water, muscle mass and serum albumin level. The distribution volume of water soluble drugs is decreased leading to their potential toxicity, while the distribution volume of lipid soluble drugs is increased leading to prolongation of elimination half-life and accumulation of the drug in fatty tissues. The drug metabolism is influenced by decreased hepatic blood flow and low activity of liver enzymes. The elimination route is limited due to reduction in renal clearance $[7,8]$. Pharmacodynamic changes involve altered sensitivity to many pharmacological agents, increased sensitivity in psychotropic or cardiovascular drugs, in contrast with decreased capacity to respond to physiological challenges and side effects of the drug therapy, e.g., orthostatic hypotension [1, 9].

Extrinsic factors include fall risks related to the environment where individuals live, e.g., how medicines are taken at home $[6,10]$. Prescribed medication is an important and potentially underappreciated contributor to falls [11, 12]. The older adults are susceptible to polypharmacy and higher risk of falls [13]. Polypharmacy can increase the risk of medication-related falls, especially when at least one established fall risk-increasing drug is part of the patient's daily regimen [14]. A leading mechanism for increased risk can be sedation which slows reaction time, orthostatic hypotension, syncope, dizziness, drowziness, and blood pressure change or impaired balance [15]. Normally, long-acting medications pose a higher risk, but the metabolism and half-life of otherwise short-acting drugs can be also prolonged in older adults [16, 17]. Previous studies have presented various methods reducing fall risks in older adults, such as supplementation of vitamin D and calcium, cataract surgery, hip protectors, modification of hazard environment and adjustment of medications [18, 19]. In addition, stability of the human body is a crucial factor for reducing falls [20]. Recommended actions to improve stability include exercise programs focusing on balance and muscle strength [21]. There is a good evidence for reducing medication-induced falls. In psychotropics, the fall risk can be reduced by prospective management of adverse effects such as drowsiness, dizziness, slow reaction time and orthostatic hypotension [22, 23]. Drugs reducing blood pressure are associated with fall risk because of their hypotension effect. Thus, adjusting anti-hypertensive medication may reduce syncope and falls [24, 25]. The fall risk prevention with anticoagulants should focus on bleeding events such as a cerebral hemorrhage and associated falls [26]. Many drugs disturb vision and vestibular system, which exacerbate gait disturbance in older adults [27, 28]. In case of falls, reduced bone mineral density and osteoporosis can increase risk of fracture and more severe consequences [29].

Evidence on medication-related fall risks is not always integrated into multifactorial risk assessments which contribute to preventive programmes for reducing falls $[30,31]$. Incorporating these risks could improve accuracy of existing tools routinely used in geriatric care units as many of the current workflows do not contain medication as a risk factor [32]. This study aimed to provide a better screen on falls in institutions by developing a preliminary categorization of fall-risk-increasing drugs (FRIDs) that could be added as a risk factor to the existing fall risk assessment tool routinely used in geriatric care units.

\section{Methods}

\section{Patient data}

The patient data used in this study were retrospectively derived from two different types of health care institutions, i.e., a hospital and a nursing home in Brno, Czech Republic. Medication data on all patients who had fallen at least once during their stay at a health care institution (hospital or nursing home) within a 2-year observational period were derived from patient records. The fall is defined by World Health Organization as an event which results in a person coming to rest inadvertently on the ground or floor or other lower level [31]. The falls during a stay at a health care institution were documented as undesirable events in a protocol, which contained information about circumstances of the fall such as a description of fall, exact time and place, and consequences, e.g., severe injuries, bone fracture or head injury, bruises or bleeding risk. Descriptive and demographic information on patients (sex, age, length of stay, MMSE score and medication use) were collected from the patient 
records. Inclusion criteria of the study participants were 1) age $\geq 60$ years old, 2) evidence of at least one fall during their stay in a geriatric care unit, 3) evidence of fall documented by health care professionals (nurses, physicians). Exclusion criteria were 1) age $<60$ years old, 2) absence of falls during their stay in a geriatric care unit, 3) no evidence of falls documented by health care professionals. The nursing home had less descriptive records of their residents, e.g., they missed a cognitive impairment scale.

\section{Developing categorization of medicines increasing fall risk (FRIDs)}

The medication-related fall risk was determined by categorizing all medicines patients used according to each medicine's fall risk. The criteria for this categorization was derived from the staturory summaries of product characteristics (SmPCs) approved by authorities as part of marketing authorization within European Union countries [33]. The SmPC information of each medicine was systematically reviewed by two researchers (JM, KV) to derive the fall risk. We applied information on a direct connection between the fall risk and adverse drug effects (ADEs) disturbing the patient's balance as presented in SmPCs. This information was used to create an A, B, C medication-related fall risk categorization. Usually the mechanism leading to medication-related fall risk is one or more of the following adverse drug effects: sedation, orthostatic hypotension, syncope, dizziness, drowsiness, changes in blood pressure or impaired balance [34, 35]. All these adverse drug effects have a negative influence on a patient's balance, which can then predispose the patient to falls [36].

Adverse drug effects posing fall risk are classified in the SmPC as very common $(\geq 1 / 10)$, common $(\geq 1 / 100)$, uncommon $(\geq 1 / 1000)$, rare $(\geq 1 / 10000)$ and very rare $(<1 / 10000)$ (Table 1). Medicines with at least one of the above-mentioned adverse drug effect frequencies of "very common" or "common" in the SmPC were categorized as high fall risk medicines (category A). Similarly, the medicines with an adverse drug effect frequency "uncommon" were categorized as moderate fall risk medicines (risk level B) and those with rare or very rare frequency as no fall risk medicines (risk level C). The high-risk category A medicines are considered as a predisposition factor to fall, because the occurrence of adverse drug effects connected to falls is documented by at least one patient per each 100 patients using the same active substance. The risk information provided in the SmPC of the brand product used by the patients involved in the study was validated by comparing the information with the SmPC information of another similar product on the market.

\section{Frequency of use of high fall risk medicines in study participants with a fall history}

The second phase of the study focused on counting the frequency of use of high fall risk medicines in study participants with a fall history in institutions. This phase made use of the existing routine practice at the time of the study of assessing and scoring all patients for fall risk by nurses in the routine admission procedure. Both institutions used the same set of 4 risk items other than medications to estimate fall risk (Table 2). The original Morse Fall Score [37] had been shortened to a timesaving procedure of 4 items suitable for clinical practice. Each fall risk item yielded 1 point and they assessed 1) history of falls, 2) mental condition, 3) physical condition, and 4) occurrence of dizziness or drowsiness in older patients. The estimation was based on patient interviews on admission or notes from patient records. Direct questions to the patient were dependent on the patient's health condition. As this fall risk estimation did not consider patient's medication as a contributor to the fall risk, the patient data on medication use were retrospectively collected from the patient records by the researcher (JM). All the medicines were classified into three risk categories (high, moderate and none) using the new categorization based on the fall risk information in the SmPCs. For the descriptive feasibility testing, the medication data of the patients in the hospital and nursing home were combined to provide the widest possible coverage of medicines used by older adults in institutions.

Table 1 Fall risk level criteria: categorization according to information derived from the summaries of product characteristics (SmPC) on the frequency of adverse drug effects (ADEs) contributing to fall risk (sedation, orthostatic hypotension, syncope, dizziness, drowsiness, changes in blood pressure or impaired balance)

\begin{tabular}{lll}
\hline Frequency of ADEs & Number of patients experiencing ADEs & Fall risk level \\
\hline Very common & $\geq 1 / 10$ & A \\
Common & $\geq 1 / 100$ to $<1 / 10$ & A \\
Uncommon & $\geq 1 / 1000$ to $<1 / 100$ & B \\
Rare & $\geq 1 / 10000$ to $<1 / 1000$ & C \\
Very rare & $<1 / 10000$ & C \\
\hline
\end{tabular}


Table 2 The items included in the existing fall risk estimation routinely used in the two health care institutions participating in the study (a hospital and a nursing home)

\begin{tabular}{llll}
\hline Fall risk item & Responsible person & Confirmation & Points in the scale \\
\hline Evidence of fall during a month prior the assessment & nurse & $\checkmark$ & 1 \\
Confusion, lack of risk perception, restlessness & nurse & $\checkmark$ & 1 \\
Impaired balance, gait disturbance & nurse & $\checkmark$ & 1 \\
Occurrence of dizziness and drowsiness & nurse & 1 \\
\hline
\end{tabular}

\section{Statistical analysis}

Descriptive statistics were used in both study phases to categorize active substances according to their fall risk, and to test the categorizations with a retrospective sample of patients from a secondary care hospital and a nursing home. All patients were grouped together for the analysis. Data were processed in Microsoft Excel, version 2016. A pivot table was created to analyze worksheet data. Descriptive statistics were used for patient data to count metric items such as frequencies, means, ranges, and standard deviations. The categorization of medicines according to their fall risk used ATC codes, specified in levels of anatomical main group, therapeutic subgroup, and pharmacological subgroup [38]. The commonly used active substances in the high fall risk category A were processed from the retrospective patient data as the frequency of use.

\section{Results}

\section{Study participants}

In total 188 fall-experienced older patients were retrospectively drawn from the patient registers of both institutions included in this study covering the 2-year period from January 2016 to December 2017 (Table 3). Of the patients, 128 (68\%) were from the geriatric department of a secondary care hospital and 60 (32\%) from a nursing home. Of them, 103 were females and 85 males with the mean age of 79 years (range: 60-97 years, standard deviation \pm 18.5 ). They used on average 9.3 medicaments (range: $2-17$, standard deviation \pm 7.5 ) during a stay in a health care institution. The mean length of stay of the study patients in the secondary care hospital $(n=128)$ was 15.8 days (range: $1-56$ days, standard deviation \pm
27.5). The most common causes of hospitalization were cardiovascular problems, and/or respiratory and urogenital tract infections. The half (52\%) of the patients had at least a mild cognitive impairment ( $\leq 23$ points), the mean score in the Mini Mental State Exam (MMSE) being 21.7 points (range: $5-30$ points, standard deviation \pm 12.5 ). The stay for nursing home residents $(n=60)$ was permanent. Data about their cognitive function was not available. They suffered from many chronic diseases (cardiac, neurologic) and used chronic long-term medications.

\section{The fall risk scores of study participants assessed on admission}

Medications were not included in the routinely used assessment. Nurses had assessed and scored all patients $(n=188)$ for fall risk on admission using the original 4 risk items (score range 0-4). This fall risk assessment is a part of the regular procedure in each patient admission. More than half $(53 \%, n=100)$ of the patients were scored as having at least high fall risk (had 3 or 4 risk scores), the remaining patients $(46 \%, n=88)$ being scored with medium or lower risk (Table 4).

\section{Categorization of medicines according to their fall risk by applying SmPCs and frequency of use}

Fall-experienced patients $(n=188)$ used altogether 1748 medicaments. Of these medicaments, 710 (41\%) belonged to the category of high fall risk (category A), 331 (19\%) moderate risk (category B) and 707 (40\%) no risk (category $\mathrm{C}$ ). The mean number of high-risk medications (category A) in use per patient was 3.8 medications, minimum $0 \quad(n=2)$, maximum $8 \quad(n=4)$ and median being $3(n=46)$. The majority $(93 \%)$ of high-risk category A medicines $(n=710)$ used by the patients were

Table 3 Characteristics of the participants from two health care institutions included in the study $(n=188$, of which hospital inpatients, $n=128$; nursing home residents, $n=60$ )

\begin{tabular}{lllll}
\hline Characteristics & & Hospital & Nursing home & Total \\
\hline Sex $\mathrm{n}(\%)$ & Male & $67(52)$ & $18(30)$ & $85(45)$ \\
& Female & $61(48)$ & $42(70)$ & $103(55)$ \\
Age, years, mean (range) & & $80(60-97)$ & $77(63-95)$ & $79(60-97)$ \\
MMSE, mean (range) & & $21.7(5-30)$ & unknown & - \\
Length of stay, days, mean (range) & & $15.8(1-56)$ & permanent & - \\
Number of drugs in use, mean (range) & $10.0(3-17)$ & $7.8(2-15)$ & $9.3(2-17)$ \\
\hline
\end{tabular}


Table 4 Distribution of fall risk scores in fall-experienced patients $(n=188)$ using 4 fall risk items routinely assessed by nurses on admission (each item yielding 1 point, score range 0-4). Medications were not included in the assessement

\begin{tabular}{llll}
\hline Scores & Fall risk level & Number of patients & Proportion (\%) of patients \\
\hline $\mathbf{0}$ & No risk & 5 & 3 \\
$\mathbf{1}$ & Low risk & 17 & 9 \\
$\mathbf{2}$ & Medium risk & 66 & 35 \\
$\mathbf{3}$ & High risk & 69 & 37 \\
$\mathbf{4}$ & Full risk & 31 & 16 \\
\hline
\end{tabular}

for the nervous system $(53 \%, n=375)$ and the cardiovascular system $(40 \%, n=281)$ (Fig. 1). The nervous system medicines in use were mainly psychotropic medications (73\% of the medicines in use in this category), while analgesics (16\%), antiepileptics (8\%) and antiparkinson drugs $(3 \%)$ represented a minority. Psychotropic medications $(n=274)$ consisted of 1$)$ psycholeptics $(n=179$, representing $65 \%$ of the medicines in this category) such as antipsychotics $(n=109,40 \%)$, anxiolytics $(n=55$, $20 \%)$, hypnotics and sedatives $(n=15,5 \%)$, and 2$)$ psychoanaleptics $(n=95$, representing $35 \%$ of the medicines in this category) such as antidepressants $(n=65,24 \%)$ and anti-dementia drugs $(n=30,11 \%)$. The high fall risk cardiovacular medications $(n=281)$ included agents acting on the renin-angiotension system (RAS) $(n=92,33 \%$ of the medicines in use in this category), beta blocking

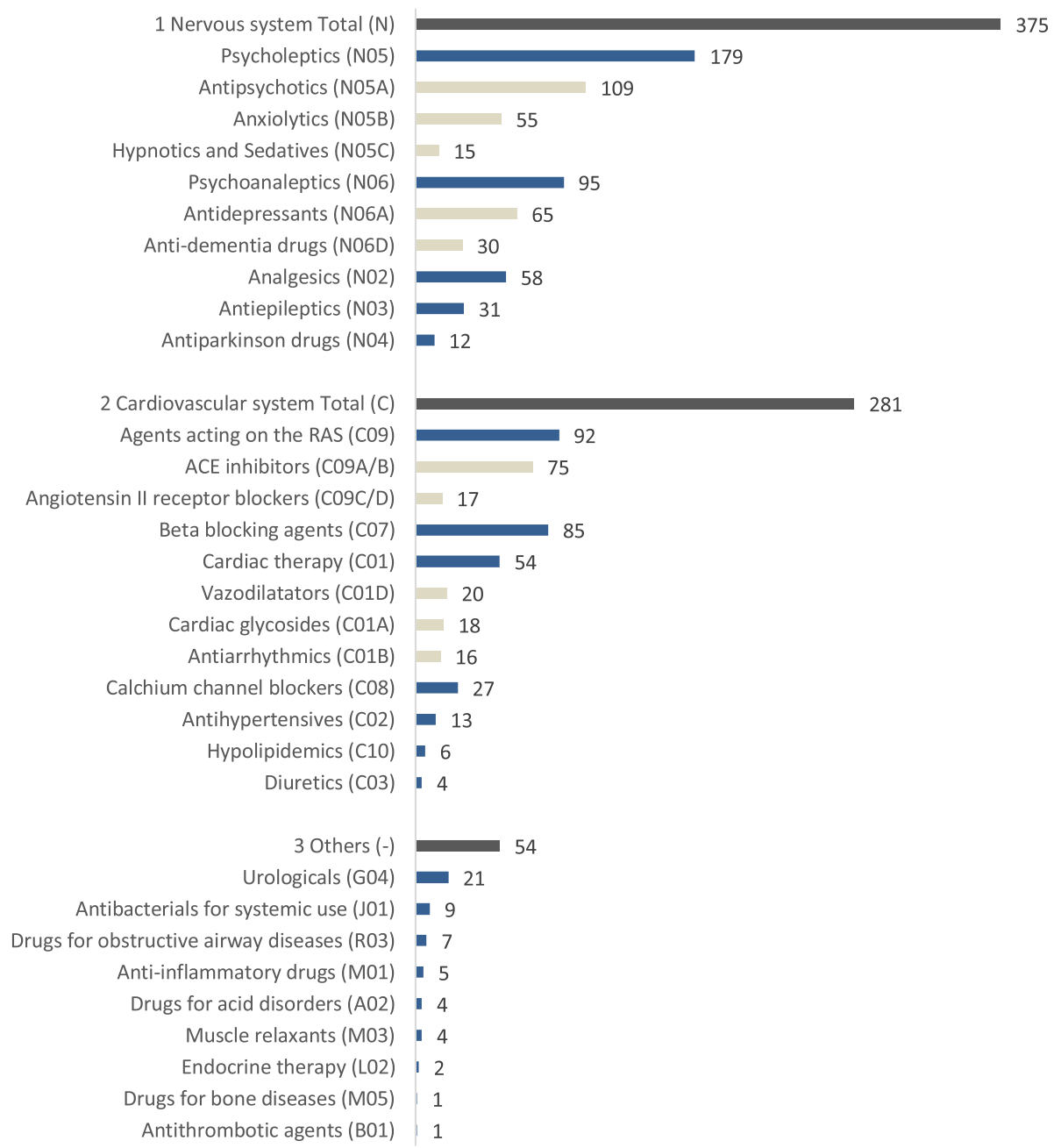

Fig. 1 Categorization of high fall-risk Category A medicines $(n=710)$ according to therapeutic group and number of users. The medicines are organized according to the ATC Classification [38].. Anatomical main group (1st level, dark grey), Therapeutic subgroup (2nd level, blue), Pharmacological subgroup (3rd level, grey) 
agents $(n=85,30 \%)$, cardiac therapy $(n=54,19 \%)$, calcium channel blockers $(n=27,10 \%)$ and other therapeutic subgroups $(n=23,8 \%)$.

Of the 216 active substances used by the patients, 102 (47\%) were classified in the high-risk category (fall risk level A), 29 (13\%) in the moderate risk category (fall risk level B) and 85 (40\%) in no risk category (fall risk level C). The leading mechanisms of ADEs consequencing in high fall risk of most commonly used active ingredients by study participants are presented in Table 5 . The most commonly used active ingredients in the high fall risk category A $(n=$ $710)$ are presented in Table 6, included metoprolol $(n=49$, beta blocking agents), perindopril ( $n=38$, ACE inhibitors), tiapride $(n=36$, antipsychotics), quetiapine $(n=24$, antipsychotics), tramadol ( $\mathrm{n}=24$, opioids), citalopram ( $n=23$, antipressants), amlodipine $(\mathrm{n}=23$, calcium channel blockers), melperone $(\mathrm{n}=23$, antipsychotics), bromazepam ( $n=20$, anxiolytics), digoxin $(n=18$, cardiac glycosides), and ramipril ( $\mathrm{n}=18$, ACE inhibitors).

\section{Discussion}

This study developed a preliminary categorization to identify medicines that may increase a fall risk in older adults in institutions, by using evidence derived from statutory medicines information and retrospective patient records. Our results indicate that numerous medicines have such an ADE profile that can lead to an increased fall risk and that the use of these high fall-risk medicines, even concomitant use of several active ingredients, is common in older adults during a hospital stay or while living in a nursing home. These findings suggest incorporating a medication-related fall risk indicator in a routine fall risk assessment on admission to geriatric care units. In long-term care units such as nursing homes, it may be useful to repeat the fall risk assessment regularly, e.g., at least once a year.

The high-fall risk categorization presented in this study considered also patient data which gives an idea of the prevalence of clinical use of different medicines. By this way it was possible to identify a few widely used medicines with a high fall risk. The top three clearly most commonly used high fall risk active substances were metoprolol (beta blocking agent), perindopril (ACE inhibitor) and tiapride (antipsychotic agent). Of the top ten active ingredients, $6 / 10$ were psychotropics (3 of them antipsychotics) and 4/10 cardiovascular agents. Optimizing the use of these few medicines in terms of prospectively managing fall risk could make a remarkable change in the incidence of actual falls in geriatric care units.

Our findings concerning the "ranking "of drugs with a high fall risk are in line with previous research. The association with falls has been consistently reported for psychotropic and cardiovascular medicines [3945]. The recent systematic reviews and meta-analyses (Seppala [43] and de Vries [44]) confirmed the association between certain drug classes and fall risk from psychotropics, i.e., antidepressants $(\mathrm{OR}=1.57)$, antipsychotics $(\mathrm{OR}=1.54)$, and benzodiazepines $(\mathrm{OR}=$ 1.42), and cardiovascular drugs, i.e., cardiac glycosides $(\mathrm{OR}=1.6)$, antiarrhythmics $(\mathrm{OR}=1.27)$, vazodilatators $(\mathrm{OR}=1.03)$, and $\mathrm{ACE}$ inhibitors $(\mathrm{OR}=1.03)$. The increased fall risk is also evidenced in patients using opioids $(\mathrm{OR}=1.61)$, antiepileptics $(\mathrm{OR}=1.55)$, antiParkinson drugs $(\mathrm{OR}=1.54)$, and NSAIDs $(\mathrm{OR}=1.09)$

Table 5 The most commonly used fall risk increasing drugs (Category A) having very common ( $\geq 1 / 10)$ or common ( $\geq 1 / 100$ ) frequency of ADEs connected to fall risk according to statutory summary of product characteristics (SmPCs)

\begin{tabular}{|c|c|c|}
\hline Active ingredient (number of users) & Drug class (ATC) & Frequency of ADEs according to SmPCs \\
\hline Metoprolol $(n=49)$ & Beta blocking agents (CO7A) & Common: dizziness, bradycardia \\
\hline Perindopril $(n=38)$ & ACE inhibitors (CO9A) & Common: dizziness, orthostatic hypotension, syncope \\
\hline Tiapride $(n=36)$ & Antipsychotics (N05A) & Common: dizziness, drowsiness \\
\hline Quetiapine ( $n=24)$ & Antipsychotics (N05A) & $\begin{array}{l}\text { Very common: dizziness, drowsiness } \\
\text { Common: orthostatic hypotension }\end{array}$ \\
\hline Tramadol $(n=24)$ & Opioids (N02A) & $\begin{array}{l}\text { Very common: dizziness } \\
\text { Common: drowsiness }\end{array}$ \\
\hline Citalopram $(n=23)$ & Antidepressants (N06A) & $\begin{array}{l}\text { Very common: drowsiness } \\
\text { Common: dizziness }\end{array}$ \\
\hline Amlodipine $(n=23)$ & Calcium channel blockers (C08C) & Common: dizziness, drowsiness \\
\hline Melperon $(n=23)$ & Antipsychotics (N05A) & $\begin{array}{l}\text { Very common: sedation } \\
\text { Common: dizziness }\end{array}$ \\
\hline Bromazepam $(n=20)$ & Anxiolytics (N05B) & Common: drowsiness, falls ${ }^{a}$ \\
\hline Digoxin $(n=18)$ & Cardiac glycosides (C01A) & Common: bradycardia, dizziness \\
\hline Ramipril ( $n=18)$ & ACE inhibitors (CO9A) & Common: dizziness, orthostatic hypotension, syncope \\
\hline
\end{tabular}

anknown frequency of $A D E$ 
Table 6 The preliminary categorization of high fall risk medicines (Category A) basing on their adverse drug effect (ADE) profiles in statutory summaries of product characteristics (SmPCs) and frequency of use in older patients who had experienced at least one documented fall in a geriatric care unit $(n=188)$. The medicines are organized according to the ATC Classification [38]

\begin{tabular}{|c|c|c|c|}
\hline High fall risk $(A)$ & ATC Code & ATC classification & Users of the medicines $\mathrm{n}(\%)$ \\
\hline Metoprolol & C07AB02 & Beta blocking agents & $49(26.1)$ \\
\hline Perindopril & C09AA04 & ACE inhibitors & $38(20.2)$ \\
\hline Tiapride & N05AL03 & Antipsychotics & $36(19.1)$ \\
\hline Quetiapine & N05AH04 & Antipsychotics & $24(12.8)$ \\
\hline Tramadol & N02AX02 & Opioids & $24(12.8)$ \\
\hline Citalopram & N06AB04 & Antidepressants & $23(12.2)$ \\
\hline Amlodipine & C08CA01 & Calcium channel blockers & $23(12.2)$ \\
\hline Melperone & N05AD03 & Antipsychotics & $23(12.2)$ \\
\hline Bromazepam & N05BA08 & Anxiolytics & $20(10.6)$ \\
\hline Ramipril & C09AA04 & ACE inhibitors & $18(9.6)$ \\
\hline Digoxin & C01AA05 & Cardiac glycosides & $18(9.6)$ \\
\hline Tramadol combination & N02AJ13 & Opioids & $16(8.5)$ \\
\hline Bisoprolol & C07AB07 & Beta blocking agents & $16(8.5)$ \\
\hline Zolpidem & N05CF02 & Hypnotics and Sedatives & $15(8.0)$ \\
\hline Donepezil & N06DA02 & Anti-dementia drugs & $14(7.4)$ \\
\hline Mirtazapine & N06AX11 & Antidepressants & $14(7.4)$ \\
\hline Amiodarone & C01BD01 & Antiarrhythmics & $14(7.4)$ \\
\hline Oxazepam & N05BA04 & Anxiolytics & $14(7.4)$ \\
\hline Tamsulosin & G04CA02 & Urologicals & $14(7.4)$ \\
\hline Memantine & N06DX01 & Anti-dementia drugs & $12(6.4)$ \\
\hline Diazepam & N05BA01 & Anxiolytics & $11(5.9)$ \\
\hline Gabapentin & N03AX12 & Antiepileptics & $11(5.9)$ \\
\hline Trazodone & N06AX05 & Antidepressants & $10(5.3)$ \\
\hline Isosorbide mononitrate & C01DA14 & Vasodilatators & $10(5.3)$ \\
\hline Clonazepam & N03AE01 & Antiepileptics & $10(5.3)$ \\
\hline Codein combination & N02AJ06 & Opioids & $9(4.8)$ \\
\hline Risperidone & N05AX08 & Antipsychotics & $8(4.3)$ \\
\hline Losartan & C09CA01 & AT II receptor blockers & $8(4.3)$ \\
\hline Sertraline & N06AB06 & Antidepressants & $7(3.7)$ \\
\hline Levodopa & N04BA01 & Dopaminergic agents & $7(3.7)$ \\
\hline Aminophylline & R03DA05 & Drugs for obstructive airway diseases & $7(3.7)$ \\
\hline Carvedilol & C07AG02 & Beta blocking agents & $7(3.7)$ \\
\hline Fentanyl & N02AB03 & Opioids & $6(3.2)$ \\
\hline Rosuvastatin & C10AA07 & Lipid modifying agents & $6(3.2)$ \\
\hline Betaxolol & C07AB05 & Beta blocking agents & $6(3.2)$ \\
\hline Haloperidol & N05AD01 & Antipsychotics & $6(3.2)$ \\
\hline Levomepromazine & N05AA02 & Antipsychotics & $6(3.2)$ \\
\hline Pregabalin & N03AX16 & Antiepileptics & $6(3.2)$ \\
\hline Nitrofurantoin & J01XE01 & Antibacterials for systemic use & $6(3.2)$ \\
\hline Urapidil & C02CA06 & Antiadrenergic agents & $5(2.7)$ \\
\hline Rilmenidine & C02AC06 & Antiadrenergic agents & $5(2.7)$ \\
\hline Nebivolol & C07AB12 & Beta blocking agents & $5(2.7)$ \\
\hline
\end{tabular}


Table 6 The preliminary categorization of high fall risk medicines (Category A) basing on their adverse drug effect (ADE) profiles in statutory summaries of product characteristics (SmPCs) and frequency of use in older patients who had experienced at least one documented fall in a geriatric care unit $(n=188)$. The medicines are organized according to the ATC Classification [38] (Continued)

\begin{tabular}{|c|c|c|c|}
\hline High fall risk (A) & ATC Code & ATC classification & Users of the medicines $\mathrm{n}(\%)$ \\
\hline Escitalopram & N06AB10 & Antidepressants & $5(2.7)$ \\
\hline Telmisartan & C09CA07 & AT II receptor blockers & $5(2.7)$ \\
\hline Midazolam & N05CD08 & Hypnotics and Sedatives & $5(2.7)$ \\
\hline Alprazolam & N05BA12 & Anxiolytics & $5(2.7)$ \\
\hline Perindopril/Indapamide & CO9BA04 & ACE inhibitors combination & $5(2.7)$ \\
\hline Verapamil & C08DA01 & Calcium channel blockers & $4(2.1)$ \\
\hline Amiloride/Hydrochlorthiazide & C03EA01 & Diuretics & $4(2.1)$ \\
\hline Rivastigmine & N06DA03 & Anti-dementia drugs & $4(2.1)$ \\
\hline Olanzapine & N05AH03 & Antipsychotics & $4(2.1)$ \\
\hline Dutasteride/Tamsulosin & G04CA52 & Urologicals & $4(2.1)$ \\
\hline Trimetazidine & C01EB15 & Other cardiac preparations & $4(2.1)$ \\
\hline Fosinopril & C09AA09 & ACE inhibitors & $3(1.6)$ \\
\hline Isosorbide dinitrate & C01DA08 & Vasodilatators & $3(1.6)$ \\
\hline Aceclofenac & M01AB16 & Anti-inflammatory drugs & $3(1.6)$ \\
\hline Captopril & C09AA01 & ACE inhibitors & $3(1.6)$ \\
\hline Baclofen & M03BX01 & Muscle relaxants & $3(1.6)$ \\
\hline Lansoprazole & A02BC03 & Drug for peptic ulcer and reflux & $3(1.6)$ \\
\hline Cefuroxime & J01DC02 & Antibacterials for systemic use & $3(1.6)$ \\
\hline Levetiracetam & N03AX14 & Antiepileptics & $2(1.1)$ \\
\hline Propafenone & C01BC03 & Antiarrhythmics & $2(1.1)$ \\
\hline Carbamazepine & N03AF01 & Antiepileptics & $2(1.1)$ \\
\hline Diclofenac & M01AB05 & Anti-inflammatory drugs & $2(1.1)$ \\
\hline Solifenacin/Tamsulosin & G04CA53 & Urologicals & $2(1.1)$ \\
\hline Oxycodone & N02AA05 & Opioids & $2(1.1)$ \\
\hline Trandolapril & C09AA10 & ACE inhibitors & $2(1.1)$ \\
\hline Levodopa/Carbidopa & N04BA02 & Dopaminergic agents & $2(1.1)$ \\
\hline Perindopril/Amlodipine/Indapamide & C09BX01 & ACE inhibitors combination & $2(1.1)$ \\
\hline Bicalutamide & L02BB03 & Hormone antagonists & $2(1.1)$ \\
\hline Glyceryl trinitrate & C01DA02 & Vasodilatators & $2(1.1)$ \\
\hline Fluoxetine & N06AB03 & Antidepressants & $1(0.5)$ \\
\hline Dosulepin & N06AA16 & Antidepressants & $1(0.5)$ \\
\hline Mianserin & N06AX03 & Antidepressants & $1(0.5)$ \\
\hline Candesartan & C09CA06 & AT II receptor blockers & $1(0.5)$ \\
\hline Venlafaxine & N06AX16 & Antidepressants & $1(0.5)$ \\
\hline Telmisartan/Hydrochlorothiazide & C09DA07 & AT II receptor blockers combination & $1(0.5)$ \\
\hline Tizanidine & M03BX02 & Muscle relaxants & $1(0.5)$ \\
\hline Lisinopril & C09AA03 & ACE inhibitors & $1(0.5)$ \\
\hline Famotidine & A02BA03 & Drug for peptic ulcer and reflux & $1(0.5)$ \\
\hline Quinapril/Hydrochlorothiazide & C09BA06 & ACE inhibitors combination & $1(0.5)$ \\
\hline Naftidrofuryl & C04AX21 & Peripheral vasodilatators & $1(0.5)$ \\
\hline Celiprolol & C07AB08 & Beta blocking agents & $1(0.5)$ \\
\hline Paroxetine & N06AB05 & Antidepressants & $1(0.5)$ \\
\hline
\end{tabular}


Table 6 The preliminary categorization of high fall risk medicines (Category A) basing on their adverse drug effect (ADE) profiles in statutory summaries of product characteristics (SmPCs) and frequency of use in older patients who had experienced at least one documented fall in a geriatric care unit $(n=188)$. The medicines are organized according to the ATC Classification [38] (Continued)

\begin{tabular}{llll}
\hline High fall risk (A) & ATC Code & ATC classification & Users of the medicines n (\%) \\
\hline Methyldopa & CO2AB01 & Antiadrenergic agents & $1(0.5)$ \\
Tianeptine & N06AX14 & Antidepressants & $1(0.5)$ \\
Doxazosin & C02CA04 & Antiadrenergic agents & $1(0.5)$ \\
Ticagrelor & B01AC24 & Antithrombotic agents & $1(0.5)$ \\
Losartan/Hydrochlorothiazide & C09DA01 & ACE inhibitors combination & $1(0.5)$ \\
Codeine & R05DA04 & Cough suppressants & $1(0.5)$ \\
Acebutolol & C07AB04 & Beta blocking agents & $1(0.5)$ \\
Mirabegron & G04BD12 & Urologicals & $1(0.5)$ \\
Ibandronic acid & M05BA06 & Drugs affecting bone & $1(0.5)$ \\
Levodopa/Benserazide & N04BA02 & Dopaminergic agents & $1(0.5)$ \\
Selegiline & N04BD01 & Dopaminergic agents & $1(0.5)$ \\
Moxonidine & C02AC05 & Antiadrenergic agents & $1(0.5)$ \\
Irbesartan & C09CA04 & ACE inhibitors & $1(0.5)$ \\
Perindopril/Amlodipine & C09BB04 & ACE inhibitors combination & $1(0.5)$ \\
Cilazapril & C09AA08 & ACE inhibitors & $1(0.5)$ \\
Fluphenazine & N05AB02 & Antipsychotics & $1(0.5)$ \\
Ropinirole & N04BC04 & Dopaminergic agents & $1(0.5)$ \\
Chlorprothixene & N05AF03 & Antipsychotics & $1(0.5)$ \\
\hline
\end{tabular}

$[46,47]$. Furthermore, the previous study from Czech Republic (Maly et al. [48]) classified drugs that affected to the nervous system (antipsychotics, antidepressants, analgesics) and to the cardiovascular system (diuretics, beta blocking agents, agents acting on the renin-angiotensin system) as the most frequently used fall risk-increasing drugs in hospitals. Thus, many studies have reached similar conclusions, although the fall-increasing medicines have been presented slightly in different order. The identification of increased risk of falls in different drug classes might be a crucial risk factor for falls. The European Geriatric Medicine Society and Finnish Expert Group on Fall-Risk-Increasing Drugs (FRIDs) have concluded that the knowledge about the risk of falls associated with therapeutic classes and individual medications can help in fall prevention [49].

According to our findings, the fall risk assessment tool presented in this study could be helpful to prevent medication-related falls and increase quality of geriatric care in health care institutions. This is supported by the findings of previous studies that have reported the medication use as a remarkable but modifiable fall risk factor $[48,50]$. Therefore, it is reasonable to implement a strategy to avoid use of fall risk-increasing drugs in the routine practice in geriatric care units. Nevertheless, many of developed fall risk assessment tools do not consider medication as a risk factor included in the tool. A review of twenty fall risk assessment tools found that only seven of the tools consider medication use as a risk factor [32]. More recent tools include medication use in a fall risk assessment, e.g., The Johns Hopkins Fall Risk Assessment Tool: it ranks opiates, anticonvulsants, antihypertensives, diuretics, hypnotics, laxatives, sedatives, and psychotropics among high fall risk drugs [51].The Johns Hopkins Fall Risk Assessment Tool is wellstructured and validated, but the total number of risk points to evaluate is high (maximum 28). This makes it more time consuming for health care providers to assess the fall risk than by using a tool with fewer risk points, such as the five items suggested to be used in our updated fall risk assessment tool. Our tool is also unique in the way that the list of fall risk medicines was created basing on statutory medicines information presented in the summaries of product characteristics approved by European Union (EU) authorities.

This study indicates that the current practice of fall risk estimation in geriatric care units without including medications underestimates the actual fall risk. More than half $(53 \%, n=100)$ of the patients included in our study scored at least a high fall risk, but the remaining $46 \%(n=88)$ of these fall-experienced patients scored a medium risk (35\%), a low risk (9\%) or no risk (3\%). The addition of a new item scoring the exposure to the high fall risk medication could refine the prospective risk assessments. This new risk item represented by high fall risk medicines (FRIDs) should 
be considered in each patient admission or medication change and periodically assessed in the existing fall risk assessment tool by health care experts.

Fine-tuning the fall risk scoring system requires further research and comparisons with the contents of other existing tools. Further research should compare how much adding high risk medicines to the fall risk assessment tool changes the risk score and makes the risk score more accurate in terms of predicting and preventing actual falls. It also might be useful to assess whether concomitant use of more than one FRID medicines will elevate the fall risk. Determining this in future studies may be necessary because the concomitant use of several drugs that increase the risk of falls was common in our data (on average 3.8 high risk medicaments per patient). In addition to this kind of risk verification research, more generalizable results are needed by testing the fall risk assessment tool with a larger number of geriatric patients from a larger number of hospital wards and nursing homes. These studies should be based on prospective patient data. Furthermore, the fall risk profiles and patterns of the high fall risk medicines should be further investigated in older adults in hospital and home care settings.

\section{Study limitations}

When interpreting the results of this study it is important to keep in mind that the study does not cover the complete spectrum of medicines used by older adults, only the medicines used by the 188 study patients. Furthermore, the retrospective patient and medication data from the patient records may perform as a source of bias. The data related to the nursing home residents were not documented in such detail compared to that of hospital patients. The differences in the detailedness of documentation concern e.g., information on cognitive impairment or changes made to medications of individual patients. Documentation may have missed minor falls which were not reported to the staff by the patients.

\section{Conclusion}

It was possible to develop a preliminary categorization of FRIDs basing on their adverse drug effect profile in SmPCs and frequency of use in older patients who had experienced at least one documented fall in a geriatric care unit. Even though more than half of the fallexperienced older adults had at least high fall risk scores on admission according to the fall risk items routinely assessed, their fall risk might have been underestimated as use of high fall risk medicaments was common, even concomitant use. Further studies are needed to develop the FRID categorization and assess its impact on fall risk.

\section{Abbreviations}

ADE: Adverse Drug Effect; ATC Classification: Anatomical Therapeutic Chemical Classification; EU: European Union; FRID: Fall Risk Increasing Drug; MMSE: Mini Mental State Exam; OR: Odds Ratio; SmPC: Summary of Product Characteristic

\section{Acknowledgements}

The authors would like to thank Professor Charles Alan Lyles from the University of Baltimore, USA for his valuable comments in preparing the manuscript.

\section{Authors' contributions}

Study concept and design: JM, KV. Interpretation of data: JM, KV, MA. Drafting of manuscript: JM, MA. Revision of manuscript: JM, MA, KB. All authors have read and approved the final manuscript.

\section{Funding}

Not applicable.

\section{Availability of data and materials}

The datasets derived from summaries of product characteristics during the current study are available from the corresponding author on reasonable request. The datasets generated and analysed during this study that were derived from retrospective patient records are not publicly available due to regulations on secondary use of patient data and subsequent agreements with the institutions to perform the study.

\section{Ethics approval and consent to participate}

The study protocol was approved by the University Hospital Brno, Jihlavska 20, Brno, Czech Republic with an authorization by Head of Geriatric Clinic and Assistant Director for Health Care. No ethics approval was required for this retrospective study (Ethics Committee of University Hospital Brno). An informed written consent was voluntarily signed by all study participants to use their anonymous data for education and research purposes.

Consent for publication

Not applicable.

\section{Competing interests}

All authors declare that there are no conflicts of interest.

\section{Author details}

${ }^{1}$ Faculty of Pharmacy, Department of Applied Pharmacy, Masaryk University, Palackeho 1946/1, 61242 Brno, Czech Republic. ${ }^{2}$ Faculty of Pharmacy,

Division of Pharmacology and Pharmacotherapy, Clinical Pharmacy Group, University of Helsinki, Viikinkaari 5E, 00014 Helsinki, Finland. ${ }^{3}$ Clinic of Internal Medicine, Geriatrics and Practical Medicine, University Hospital Brno, Jihlavska 20, 62500 Brno, Czech Republic

Received: 15 January 2020 Accepted: 22 October 2020

Published online: 06 November 2020

\section{References}

1. Huang AR, Mallet $L$, Rochefort CM, Eguale T, Buckeridge DL, Tamblyn R. Medication-related falls in the elderly: causative factors and preventive strategies. Drugs Aging. 2012;29(5):359-76.

2. Weil TP. Patient falls in hospitals. An increasing problem. Geriatr Nurs. 2015; 36(5):342-7

3. Tinetti ME, Kumar C. The patient who falls: it is always a trade-off. JAMA. 2010;303(3):258-66.

4. Burns Z, Khasnabish S, Hurley AC, et al. Classification of injurious fall severity in hospitalized adults. J Gerontol A Biol Sci Med Sci. 2020. https://doi.org/10. 1093/gerona/glaa004.

5. Vaapio SS, Salminen MJ, Ojanlatva A, Kivelä SL. Quality of life as an outcome of fall prevention interventions among the aged: a systematic review. Eur J Pub Health. 2009:19(1):7-15.

6. Ambrose AF, Paul G, Hausdorff JM. Risk factors for falls among older adults: a review of the literature. Maturitas. 2013;75(1):51-61.

7. Mangoni AA, Jackson SHD. Age-related changes in pharmacokinetics and phamacodynamics: basic principles and practical application. Br J Clin Pharmacol. 2003;57(1):6-14. 
8. Turnheim K. When drug therapy gets old. Pharmacokinetics and pharmacodynamics in the elderly. Exp Gerontol. 2003;38(8):843-53.

9. Wooten JM. Pharmacotherapy considerations in elderly adults. South Med J. 2012:105(8):437-45.

10. Jung $D$, Shin $S$, Kim H. A fall prevention guideline for older adults living in long-term care facilities. Int Nurs Rev. 2014:61(4):525-33.

11. Lawlor DA, Patel R, Ebrahim S. Association between falls in elderly woman and chronic diseases and drug use: cross sectional study. BMJ. 2003; 327(7417):712-7.

12. Neutel Cl, Perry S, Maxwell C. Medication use and risk of falls. Pharmacoepidemiol and Drug Saf. 2002;11(2):97-104.

13. World Health Organization. Medication Safety in Polypharmacy: Technical Report. 2019. Available at: https://apps.who.int/iris/handle/10665/325454. Accessed Sep 2020.

14. Ziere G, Dieleman JP, Hofman A, Pols HA, van der Cammen TJ, Stricker BH. Polypharmacy and falls in the middle age and elderly population. $\mathrm{Br} \mathrm{J}$ Clin Pharmacol. 2006;61(2):218-23.

15. Walgers JJ, Ruiter SC, Germans T, Kat MG, Ruiter JH, Jansen RWMM. Psychiatric symptoms and use of psychotropic medication in elderly fall and syncope patients. Eur Geriatr Med. 2017;8(5-6):419-23.

16. Ensrud KE, Blackwell TL, Mangione CM, Bowman PJ, Whooley MA, Bauer DC, Schwartz AV, Hanlon JT, Nevitt MC. Central nervous system-active medications and risk for falls in older women. J Am Geriatr Soc. 2002;50(10): 1629-37.

17. McLean AJ, Le Couteur DG. Aging biology and geriatric clinical pharmacology. Pharmacol Rev. 2004;56(2):163-84.

18. Kannus $P$, Sievanen $H$, Palvanen $M$, Jarvinen $T$, Parkkari J. Prevention of falls and consequent injuries in elderly people. Lancet. 2005;366:1885-93.

19. Kenny RA, Rubenstein LZ, Tinetti ME, et al. Summary of the updated American Geriatrics Society/British geriatrics society clinical practice guideline for prevention of falls in older persons. J Am Geriatr Soc. 2011; 59(1):148-57.

20. Tinetti ME. Preventing falls in elderly persons. N Engl J Med. 2003;348:42-9

21. Li F, Harmer P, Fischer KJ, McAuley E. Tai chi: improving functional balance and predicting subsequent falls in older persons. Med Sci Sports Exerc. 2004;36(12):2016-52.

22. Ray WA, Thapa PB, Gideon P. Benzodiazepines and the risk of falls in nursing home residents. J Am Geriatr Soc. 2000:48(6):682-5.

23. Tinetti ME, Baker DI, McAvay G, Claus EB. A multifactorial intervention to reduce the risk of falling among elderly people living in the community. N Engl J Med. 1994;331:821-7.

24. Darowski A, Whiting E. Cardiovascular drugs and falls. Rev Clin Gerontol. 2011;21(2):170-9.

25. Luiting $S$, Jansen $S$, Seppälä $\sqcup$, Daams JG, van der Velde N. Effectiveness of Cardiovaslular evaluations and interventions on fall risk: a scoping review. J Nutr Health Aging. 2019;23(4):330-7.

26. Ebadi-Robert H, Le Gal G, Righini M. Use of anticoagulants in elderly patients: practical recommendations. Clin Interv Aging. 2009;4:165-77.

27. Salonen L, Kivelä SL. Eye disease and impaired vision as possible risk factors for recurrent falls in the aged: a systematic review. Curr Gerontol Geriatr Res. 2012;12:1-10.

28. Zalewski CK. Aging of the human vestibular system. Semin Hear. 2015;36(3): 175-96

29. Edwards MH, Jameson K, Denison H, Harvey NC, Aihie-Sayer A, Dennison EM, Cooper C. Clinical risk factors, bone density and fall history in the prediction of incident fracture among men and women. Bone. 2013;52(2): 541-7.

30. Rubenstein LZ. Falls in older people: epidemiology, risk factors and strategy for prevention. Age Ageing. 2006;35(2):37-41.

31. World Health Organization. WHO Global Report on Falls Prevention in Older Age. 2008. Available at: https://www.who.int/publications-detail/who-globalreport-on-falls-prevention-in-older-age?ua=1 Accessed Sep 2017.

32. Perrel $\mathrm{KL}$, Nelson $\mathrm{A}$, Goldman $\mathrm{RL}$, et al. Fall risk assessment measures: an analytic review. J Gerontol A Biol Sci Med Sci. 2001;56(12):761-6.

33. European Commission. A Guideline on Summary of Product Characteristics. 2009. Available at: https://ec.europa.eu/health/sites/ health/files/files/eudralex/vol-2/c/smpc_guideline_rev2_en.pdf Accessed Sep 2017.

34. Glab KL, Wooding FG, Tuiskula KA. Medication-related falls in the elderly: mechanism and prevention strategies. Consult Pharm. 2014; 29(6):413-7.
35. Darowski A, Dwight J, Reynolds J. Medicines and Falls in Hospital: Guidance Sheet. 2011. Available at: https://www.shropshireccg.nhs.uk/media/2475/ guidance-sheet-medicines-and-falls-in-hospital.pdf Accessed Sep 2017.

36. Shubert TE. Evidence-based exercise prescription for balance and falls prevention: a current review of the literature. J Geriatr Phys Ther. 2001;34(3): $100-8$.

37. Morse JM. Preventing patient falls. Establishing a fall intervention program. 2nd ed. New York: Springer Publishing Company; 2009.

38. World Health Organization Collaborating Centre for Drug Statistics Methodolody. ATC/DDD Index 2019. Available at: https://www.whocc.no/ atc ddd index/ Accessed Jan 2018.

39. Leipzig RM, Cumming RG, Tinetti ME. Drugs and falls in older people: a systematic review and meta-analysis: I. Psychotropic drugs. J Am Geriatr Soc 1999;47(1):30-9.

40. Leipzig RM, Cumming RG, Tinetti ME. Drugs and falls in older people: a systematic review and meta-analysis: II. Cardiac and analgesic drugs. J Am Geriatr Soc. 1999;47(1):40-50.

41. Woolcott JC, Richardson KJ, Wiens MO, et al. Meta-analysis of the impact of 9 medication classes on falls in elderly persons. Arch of Intern Med. 2009; 169(21):1952-60.

42. Bloch F, Thibaud M, Dugue B, et al. Psychotropic drugs and falls in the elderly people: updated literature review and meta-analysis. J Aging Health. 2011;23(2):329-46

43. Seppala $L$, Wermelink AMAT, de Vries M, et al. Fall-risk-increasing drugs: a systematic review and meta-analysis: II. Psychotropics. J Am Med Dir Assoc. 2018;19(4):371.

44. de Vries M, Seppala LJ, Daams JG, et al. Fall-Risk-Increasing Drugs: A Systematic Review and Meta-Analysis: I. Cardiovascular Drugs. J Am Med Dir Assoc. 2018;19(4):371.

45. Tamblyn R, Bates DW, Buckeridge DL, et al. Multinational investigation of fracture risk with antidepressant use by class, drug, and indication. J Am Geriatr Soc. 2020. https://doi.org/10.1111/jgs.16404.

46. Seppala LJ, van de Glind EMM, Daams JG, et al. Fall-Risk-Increasing Drugs: A Systematic Review and Meta-Analysis: III. Others. J Am Med Dir Assoc. 2018; 19(4):372.

47. Yoshikawa A, Ramirez G, Smith MA, et al. Opioid use and the risk of falls, fall injuries and fractures among older adults: a systematic review and metaanalysis. J Gerontol A Biol Sci Med Sci. 2020. https://doi.org/10.1093/gerona/ glaa038.

48. Maly J, Dosedel M, Vosatka J, et al. Pharmacotherapy as major risk factor of falls - analysis of 12 months experience in hospitals in South Bohemia. J Appl Biomed. 2019;17(1):53-60

49. Seppala LJ, Velde N, Masud T, et al. EuGMS task and finish group on fall-risk-increasing drugs (FRIDs): position on knowledge dissemination, Management, and Future Research. Eur Geriatr Med. 2019;10:275-83.

50. Walsch ME, Boland F, Morarty F, Fahey T. Modification of potentially inappropriate prescribing following fall-related hospitalizations in older adults. Drugs Aging. 2019;36(5):461-70.

51. Poe SS, Cvach M, Dawson PB, et al. The Johns Hopkins fall risk assessment tool: Postimplementation evaluation. J Nurs Care Qual. 2007;22(4):293-8.

\section{Publisher's Note}

Springer Nature remains neutral with regard to jurisdictional claims in published maps and institutional affiliations.

Ready to submit your research? Choose BMC and benefit from:

- fast, convenient online submission

- thorough peer review by experienced researchers in your field

- rapid publication on acceptance

- support for research data, including large and complex data types

- gold Open Access which fosters wider collaboration and increased citations

- maximum visibility for your research: over $100 \mathrm{M}$ website views per year

At $\mathrm{BMC}$, research is always in progress.

Learn more biomedcentral.com/submission 